\section{IN BRIEF}

\section{$\Rightarrow$ RHEUMATOID ARTHRITIS}

\section{Low-dose rituximab can go even lower}

A pre-planned secondary analysis of data from the REDO trial demonstrates similar effectiveness of the ultra-low rituximab doses $1,000 \mathrm{mg}, 500 \mathrm{mg}$ and $200 \mathrm{mg}$ in patients with rheumatoid arthritis, with $11 \%, 21 \%$ and $13 \%$ of patients treated with these doses not meeting response criteria. The lower doses resulted in lower drug concentrations, but did not affect concentrations of anti-drug antibodies or counts of B cells. The lack of a dose-response suggests that even lower doses of rituximab could be effective in these patients.

ORIGINAL ARTICLE Wientjes, M. H. M. et al. Drug levels, anti-drug antibodies and B-cell counts were not predictive of response in rheumatoid arthritis patients on low dose rituximab. Rheumatology https://doi.org/10.1093/rheumatology/keac024 (2022)

\section{OSTEOARTHRITIS}

\section{PPIs linked to increased risk of knee replacement}

Proton pump inhibitors (PPIs) prescribed to counter gastrointestinal effects of NSAIDs can affect serum magnesium concentrations and, in turn, osteoarthritis $(\mathrm{OA})$ progression. In a UK study that showed an increase in PPI prescription rates in patients with knee OA from $12.7 \%$ in 2000 to $44.0 \%$ in 2017 , the risk of knee replacement was higher in patients treated with the PPIs omeprazole (HR 1.21; 95\% Cl 1.01-1.44) or pantoprazole (HR 1.38; 95\% Cl 1.00-1.90) than in patients treated with histamine 2 receptor antagonists. Treatment with lansoprazole, rabeprazole or esomeprazole was not associated with increased risk of knee replacement.

ORIGINAL ARTICLE Zeng, C. et al. Proton pump inhibitor therapy and risk of knee replacement surgery: a general population-based cohort study. Osteoarthritis Cartilage https://doi.org/10.1016/j.joca.2021.12.010 (2022)

\section{GOUT}

\section{No benefit to intensive urate lowering in gout}

Results from a 2-year, double-blind, randomized controlled trial of 104 patients with erosive gout suggest there is no benefit to assigning an intensive $(<0.2 \mathrm{mmol} / \mathrm{L})$ rather than a standard $(<0.3 \mathrm{mmol} / \mathrm{L})$ serum urate target. Patients in the intensive-target group achieved lower serum urate concentrations, but required higher doses of allopurinol and greater use of combination therapy than those in the standard-target group. The intensive-target and standard-target groups did not differ in terms of bone erosion scores, OMERACT core outcome domains or adverse event rates.

ORIGINAL ARTICLE Dalbeth, N. et al. Intensive serum urate lowering with oral uratelowering therapy for erosive gout: A randomized double-blind controlled trial. Arthritis Rheumatol. https://doi.org/10.1002/art.42055 (2021)

\section{UNDIFFERENTIATED ARTHRITIS}

\section{Imaging for prediction of RA development}

The usefulness of MRI of the hands and feet for prediction of progression to rheumatoid arthritis (RA) has been assessed in 405 patients who meet the current definition of undifferentiated arthritis by fulfilling neither the 1987 nor the 2010 criteria for RA and having no alternative diagnosis. Over a 1-year follow-up, $21 \%$ of these patients developed RA. MRI-detected synovitis and tenosynovitis were predictive of development of RA, whereas RA was less likely in individuals with negative MRI findings, particularly in the subgroup with autoantibody-negative oligoarthritis.

ORIGINAL ARTICLE den Hollander, N. K. et al. Hand and foot MRI in contemporary undifferentiated arthritis: in which patients is MRI valuable to detect rheumatoid arthritis early? - a large prospective study. Rheumatology https://doi.org/10.1093/rheumatology/ keac017 (2022)

\title{
B cells: deplete, repopulate, vaccinate
}

Immunosuppression is essential for treatment of autoimmune rheumatic disease (AIRD), but it can have negative effects on the generation of effective immune responses. B cell

depleting therapy (BCDT) is of particular concern, as it is known to affect the serological response to vaccination. With the continuing threat of the COVID-19 pandemic, rheumatologists are striving to determine how to get the best outcomes from both BCDT and SARS$\mathrm{CoV}-2$ vaccination in patients with severe AIRDs. Results from three new studies provide further evidence of the importance of the interval between rituximab treatment and vaccination, and suggest that $B$ cell reconstitution is a biomarker for the probability of seroconversion.

In a study of 24 patients with AIRD who were treated with the BCDT rituximab, 35 with AIRD and other immunosuppressant therapy and 26 healthy individuals, 28 days after second doses of SARS-CoV-2 mRNA vaccines, neutralizing antibodies were present in $29 \%, 80 \%$ and $92 \%$ of participants in the respective groups. No patient treated with rituximab in the 6 months prior to vaccination had a neutralizing antibody response, and time since last rituximab infusion was associated with humoral response. Rituximab treatment did not affect T cell responses.

In a second study of 56 patients with AIRD who were all treated with rituximab and who all received two doses of SARS-CoV-2 mRNA vaccine, time from last rituximab infusion ( $<6$ months, $6-12$ months or $>12$ months) was associated with rates of serological response (antibodies to the viral spike protein were detected in $14 \%, 45 \%$ and $87 \%$, respectively). In addition, among the 39 participants whose $B$ cell status was assessed, the seropositivity rate was $91.3 \%$ in those with detectable B cells who were vaccinated $\geq 6$ months after rituximab treatment. According to corresponding author Robert Spiera, these results "suggest that B cell measurement could provide complementary information to timing that could help inform strategies to increase the likelihood of achieving a serological response in rituximab-treated patients with AIRD."

In the third study, among 19 patients with AIRD and rituximab treatment, 12 with AIRD and other therapy and 30 healthy individuals, researchers identified the minimum concentration of B cells in the peripheral circulation of individuals who underwent seroconversion in response to SARS-CoV-2 vaccination. Corresponding author Thomas Dörner suggests that this concentration, of $10 \mathrm{~B}$ cells/ $\mu \mathrm{l}$, "is a candidate biomarker for a high likelihood of humoral vaccination response, and may support optimization of vaccination protocols among this vulnerable patient group."

Robert Phillips

ORIGINAL ARTICLES Bitoun, S. et al. Rituxima impairs B-cell response but not T-cell response to COVID-19 vaccine in auto-immune diseases. Arthritis Rheumatol. https://doi.org/10.1002/art. 42058 (2021) | Jinich, S. et al. B-cell reconstitution is strongly associated with COVID-19 vaccine responsiveness in rheumatic disease patients treated with rituximab. Arthritis Rheumatol. https://doi.org/10.1002/art.42034 (2021)| Stefanski, A. L. et al. B cell numbers predict humoral and cellular response upon SARS-CoV-2 vaccination among patients treated with rituximab. Arthritis Rheumatol. https://doi.org/ 10.1002/art.42060 (2021) 